\title{
The Effect of Configurational Entropy of Mixing on the Design and Development of Novel Materials
}

\author{
Krishanu Biswas ${ }^{1 \#}$ and Nirmal Kumar ${ }^{1}$ (ORCiD: 0000-0001-8795-7857) \\ ${ }^{1}$ Department of Materials Science and Engineering, \\ IIT Kanpur, Kanpur -28016, INDIA
}

\begin{abstract}
The configurational entropy of mixing $\left(\Delta S_{m i x}\right)$ has a profound influence on the stability of various phases in different materials at intermediate and high temperatures. Recently, it has been observed that $\Delta S_{m i x}$ can be used as an important tool to design novel multicomponent materials with fascinating properties. $\Delta S_{m i x}$ affects $\Delta G_{m i x}$ and tends to stabilize the FCC/BCC/HCP multicomponent solid solutions over brittle phases including compounds. This opens up vistas to design novel solid solution based materials with improved mechanical, functional properties. Accordingly, multicomponent and multiprinciple alloys were developed in 2004, and subsequently, novel ceramics and polymers have been designed. The present paper is intended to provide an insight into the role of $\Delta S_{\text {mix }}$ to design novel metallic, ceramic as well as polymeric materials.
\end{abstract}

Keyword: Entropy of mixing, High Entropy Alloys, Ceramics, Polymer, Design

\#Email: kbiswas@iitk.ac.in, Phone: +91-5122596184, FAX; +91-5122597505 


\section{Introduction}

The majority of the materials we use in our day-to-day life is in the alloyed form(Davis \& Committee, 1990). Alloying, the greatest gift to humankind, is defined as chemically mixing two or more components (metals, ceramics or even polymers) to obtain an atomically mixed arrangement of atoms in the crystal lattice for improvement of properties; physical, chemical, functional, mechanical, etc.(Brandes \& Brook, 1998). Therefore, the alloying has extensively been used for a long time (even during Indus Valley Civilization) to design and develop novel materials for various engineering applications (Cantor, 2014; Mohanty et al., 2015; Murty, Yeh, \& Ranganathan, 2014; Sharma, Yadav, Biswas, \& Basu, 2018; Tazuddin, Biswas, \& Gurao, 2016; Tazuddin, Gurao, \& Biswas, 2017; Yeh et al., 2004). The classical examples include steels, superalloys, brass, bronzes, mullite, silicates, etc. Alloys, in general, can be made in two basic forms, solid solutions and compounds. Solid solutions are like a liquid solution in solid state consisting of various species atomically mixed to form a crystal. On the other hand, compounds are stoichiometric compounds with small solubility range. As compared to compounds, solid solutions with simple crystal structure provide the right combination of mechanical and physical properties required to be useful in the application. Compounds, on the other hand, are strong but brittle and hence mainly find functional applications. Therefore, solid solutions having a simple crystal structure, including Face Centre Cubic (FCC), Body Centre Cubic (BCC) are considered the best materials for structural applications. The widely utilized solid solutions consisting of two or three components with one of the components as major components. These phases are stabilized by interplay between enthalpy and entropy of mixing.

\section{Configurational entropy of mixing $\left(\Delta S_{\text {mix }}\right)$}


The entropy of mixing plays its role vital the free energy of mixing of a system. It is well known that free energy of mixing $\Delta G_{m i x}=\Delta H_{m i x}-T \Delta S_{m i x}$ at any temperature $T$; where $\Delta H_{m i x}$ is the enthalpy of mixing. $\Delta H_{m i x}$ depends primarily on bond energies of the elements. In general, for elements with a strong propensity to form strong bonds, $\Delta H_{m i x}$ is considered to be negative. For elements with low or zero solubility or unlikeness to form bonds, $\Delta H_{m i x}$ is positive. However, $\Delta S_{\text {mix }}$ primarily depends on the arrangement of atoms in the crystalline lattice. For solid solutions, it is given by Boltzmann hypothesis(Gaskell, 2008),

$$
\Delta S_{\operatorname{mix}}=-k\left(\ln X_{A} X_{A}+\ln X_{B} \ln X_{B}\right)
$$

$k$ is Boltzmann constant $=R / N_{A}\left(R=\right.$ Universal gas constant, $N_{A}$ is Avogadro's number $)$ and $X_{A}$ and $X_{B}$ mole fractions of $A$ and $B$ atomic species respectively.

Hence,

$$
\Delta G_{\text {mix }}=\Delta H_{\text {mix }}-R T\left(X_{A} \ln X_{A}+X_{B} \ln X_{B}\right)
$$

For multicomponent system, i.e., $N \geq 5$,

$$
\Delta S_{\text {mix }}=-R\left(X_{A} \ln X_{A}+X_{B} \ln X_{B}+\cdots X_{E} \ln X_{E}\right.
$$

Where, $X_{A}, X_{B}, X_{C}, X_{D}$, and $X_{E}$ have usual meaning, as defined earlier.

Hence, for equimolar systems $X_{A}=X_{B}=X_{C}=X_{D}=X_{E}$

$$
\begin{aligned}
& \Delta S_{\text {mix }}=-R\left(X_{A} \ln X_{A}+\cdots X_{A} \ln X_{A}\right. \\
& =-R \ln \left(5 X_{A} \ln X_{A}\right)=R \ln 5
\end{aligned}
$$

Hence, in general, $\Delta S_{m i x}=R \ln N$ for a system having $N$ components in equimolar proportions . Thus, it is possible to estimate $\Delta S_{m i x}$ as a function of $N$. Figure 1 shows such behavior. It is 
evident that $\Delta S_{m i x}$ increases sharply for small values of $N$ and subsequently, it remains almost constant for $N=13$ or more. This provides us avenues for stabilizing solid solution by increasing number of components $(N)$ even though $\Delta H_{m i x}$ is strongly negative for elements having strong likeness to form bonds, as in case of compound formation. Therefore, it is possible to stabilize solid solution based phase having a simple crystal structure, as compared to compounds by using a large number of elements (at least 5 or more). This has important consequence and has recently been realized for use in design of novel materials, which is as discussed next. This also allows us to explore central part of the phase diagram, i.e., expand the compositional space by using larger number of component and create infinitely large number of alloy compositions, combining large number of elements in the periodic table (Inset of Fig. 1a). By adopting large number of components, unique atomic structure can be created (Fig.1b). In this figure each coloured ball denotes one atomic species and hence, atomic environment can be varied locally as compared to alloys consisting of two or three components alloys such as steels, brass, bronzes etc. Hence, this allows tuning materials property locally. The local variation of properties is effective in certain applications, especially in catalysis where catalytic activity of any atomic species can be altered locally by changing the potential of the atom. In addition, solid solution can be strengthened extensively by adopting such atomic arrangement. The solid solution hardening is primarily due to atomic size difference, creating strain field around the atom and causing motion of defects (dislocations, twins) difficult. For multi-component systems having atoms of different sizes, the barrier to the motion of the defect can be made to vary locally and hence, solid solution can extensively strengthened (Mohanty et al., 2015; Tazuddin et al., 2017). Therefore, $\Delta S_{m i x}$ allows us to divide the alloys into three categories Figure 2 shows there three regions. The conventional alloys (steels, brass, bronze, super alloy), which are based on the one or two principal elements are considered to be low entropy alloys. Since $\Delta S_{m i x} \leq 0.693 \mathrm{R}(R \ln 2)$. On 
the other hand, alloys with $N=5, \Delta S_{m i x} \geq 1.61 R$ and hence they are termed as high entropy alloys. The intermediate alloys having 2 to 4 principal elements are considered as medium entropy alloys. It is evident that the medium entropy alloys do not possess sufficient $\Delta S_{m i x}$ to stabilize solid solutions phase, leading to formation of complex and brittle intermetallic phases. From application view point, the suppression of formation of intermetallic compounds is required in order to have sufficient ductility and toughness. Hence, high $\Delta S_{m i x}$ significantly lower the free energy of the solid solution phase, leading to stabilization of these alloy phases.

The stabilization of solid solution based phases over compounds makes the multicomponent alloys a reality in the potentials application. The solid solution based phases with FCC/BCC structure can exhibit the best consideration of strength and ductility; much needed by the structural engineer to design various compounds for structural, machinery, automobile, etc. Achieving the right combination of strength, ductility and toughness are considered the holygrail in materials science and engineering. The presence of a large number of elements can provide extensive hardening due to solid solutions; which can even be tuned by proper choice of elements and composition. Secondly, it opens up a large number of choices of possible to achieve using an infinitely large combination of elements to explore the compositional space. Normally, the alloys are single or two elemental bonds and other elements have been added for the betterment of properties. Therefore, it allows the materials scientist to explore the vast composition space to design and develop different materials for various applications. The third aspect of the novel design concept involves.

Design of novel alloy (type, chemistry, specific properties start using thermodynamics is done primarily due to the fact the phase and microstructure. These play a significant role in the properties. Design is carried out by the estimation and comparing of the free energy 
change as the formation of temperature and composition. This is normally carried out using a technique is known as CALPHAD (calculation of phase diagram). This methodology has gained wider applicability due to sufficient computational power available recently. This is scientifically more sound and robust and it is able to predict phase formation for the multicomponent systems. However, such calculation for multicomponent $(n \geq 5)$ systems requires assumption to be made. CALPHAD predicts the phase based on the extrapolation and minimization (Figure 3a)(Kattner, 1997). At a given condition, the stability of phases is decided by Gibbs free energy minimization. For given conditions the stability of phases is decided by minimization of total Gibbs free energy.

Figure $3 b$ shows the results of one such five component alloy system (CoCuFeMnNi). The formation of different phases as function of temperature is shown by plotting mole fraction of phases with respect of temperature. It is evident that single phase solid solution having FCC (fcc\#1) is stable in the large temperature domain (Tazuddin et al., 2017). Only at lower temperatures $(<800 \mathrm{~K})$, some other solid solution phases (fcc\#2 and bcc) can form. Therefore, CALPHAD allows us to design various alloy systems with single phase FCC/BCC structures (Tazuddin et al., 2017).

\section{Case of multicomponent ceramics}

Recently, multicomponent entropy stabilized oxides and borides have been been reported (Rost et al., 2015). In case of the ceramics, the configurational entropy can be used to engineer novel materials by using multicomponent metallic species in the basic frames work of oxygen or boron. The metallic cations are incorporated in the octahedral voids in a novel way to increase the entropy of mixing $\left(\Delta S_{m i x}\right)$ to obtain single phase entropy -stabilized oxides or borides. These works categorically indicate that entropy of mixing in particular, predominately dictate the energy landscape, stabilizing the solid solutions. It is evident that 
the effect of entropy seems to be lower as compared to metallic sublattice (Figure 4a). The oxygen sublattice predominately order, baring same point defects. On the other hand, cationic sub lattice consisting of metallic elements provides the configurational entropy of mixing. As compared to single component oxides or borides, multicomponent based are lead to substantial increases in $\Delta S_{m i x}$, providing stabilization of the solid solution borides or oxides. In the following, the basic aspect of calculation of $\Delta S_{m i x}$ for oxide is described. For metallic systems with random solutions consisting of two elements A-B, the materials interaction energy in given by $\varepsilon_{A-B}=\varepsilon_{A-A}+\varepsilon_{B-B}$, since these is an equal probability of bond formation. Thus, all lattice sites have an equal probability of occupation and entropy is considered to be maximized. This is an ideal situation in which all interaction energies, $\varepsilon_{A-B}, \varepsilon_{A-A}, \varepsilon_{B-B}$ are equal. Any variation from the random mixture between A-B may likely to have different values of interactions energies. $\varepsilon_{A-B}, \varepsilon_{A-A}$ and $\varepsilon_{B-B}$ and hence, such formalism may not work. This situation is different in case of oxides or borides in which the metallic ions sit on the cations sublattices (Figure 4b). Here, an anion sit oxygen or boron ions at the intermediate space and hence, every cation lattices can be considered. The second nearest neighbour, the site-specific difference can also be evident. Therefore, the number of possible configuration can be maximized if each of the cationic site is considered identical as well as energetically similar. However, such consideration is not a perfect approximation as the second and higher interactions will definitely influence the possible configurations. Nevertheless, this will increase $\Delta S_{m i x}$ and hence, likely to stabilize the solid solution and lower of temperature at which entropy induced the formation of such a solid solution. It is to be noted here that, the effects of entropy on oxides system is already been investigated, i.e. cation occupancy in spinal(Navrotsky \& Kleppa, 1967), order-disorder transformation is feldspar (Megaw, 1973). However, the technique of engineering $\Delta S_{\text {mix }}$ to stabilized multicomponent oxides in a single cation sublattice could only be achieved using the principles of high entropy alloys. Any such 
efforts of exploring the vast compositional space for compound forming systems is definitely challenging, requiring both computational and experimental approaches.

Using a similar approach Gild et al. has reported the formation of entropy stabilized borides (Gild et al., 2016). These unique borides containing high melting metals [Ht,Ta,W, etc] which effectively improve the high-temperature capability of the existing materials utilized in space applications (Laura \& Diletta, 2011). Conventionally $\mathrm{TaB}_{2}, \mathrm{HfB}_{2}$ arc used for components in Space vehicles due to their unique properties including, high melting temperature low thermal conductivity low diffusivity and reactively. These properties can further be improved by engineering novel mixed borides carbides, oxides, etc (Gild et al., 2016).

\section{Case of polymers}

Polymers are anther class of materials which find extensive usage in day-to-day life. Although, there is no report of high entropy polymer till date, there has been discussion on the existence of natural polymers stabilized by entropy of mixing, akin to the metallic alloys or multicomponent ceramics. Many natural proteins are stabilized by using the strategy of maximizing the entropy of mixing. It involves allowing the change in entropy via folding/unfolding of different chain of the proteins. Such difference in the entropy between the folded and unfolded states can lead to improved thermodynamical stability of the proteins. This has intelligently been achieved by substantial increase in the configurational entropy of the folded state. Figure 5 shows model of a protein, known as hmAcP of horse muscle and a corresponding residue. The protein can contain various loop variants, altering the different states. It has been shown that entropy of mixing is given by equation(6)(Dagan et al., 2013). 


$$
\Delta S_{m i x}=c R \ln \left(n / n_{\text {ref }}\right)
$$

Here, $n$ refers to number of residues in a particular loop whereas $n_{r e f}$ is known as the number of residues in the loop of any reference mutant, $c$ is the correlation factor, connected to the persistence distance, $R$ the universal gas constant. Therefore, by making $n>>n_{r e f}$, it is possible to make $\Delta S_{m i x}$ extremely high and stabilize the protein structure. Similar approach can be extended to synthetic polymers and soon high entropy polymer can be synthesized in the lab.

\section{Conclusions}

Therefore, it has categorically been shown that the Configurational entropy of mixing $\left(\Delta S_{m i x}\right)$ has significant effect on thermodynamical stability of various phases in metallic, ceramic and polymeric systems. In fact, $\Delta \mathrm{S}_{\mathrm{mix}}$ can be effectively used as a tool to design multicomponent alloys, ceramics as well as polymers with distinct and improved properties, which would allow them to be exploited technologically. The future will unfold many interesting cases of materials development.

\section{Acknowledgments}

The authors would like to thank SERB of Department of Science and Technology, INSA and Indian Space Research Organization for funding the research carried out on various materials. 


\section{References}

Brandes, E. A., \& Brook, G. B. (1998). 3 - Mechanical properties of light metals and alloys. In E. A. B. B. Brook (Ed.), Smithells Light Metals Handbook (pp. 14-66). Oxford: Butterworth-Heinemann.

Cantor, B. (2014). Multicomponent and high entropy alloys. [Review]. Entropy, 16(9), 47494768.

Dagan, S., Hagai, T., Gavrilov, Y., Kapon, R., Levy, Y., \& Reich, Z. (2013). Stabilization of a protein conferred by an increase in folded state entropy. [Article]. Proceedings of the National Academy of Sciences of the United States of America, 110(26), 1062810633.

Davis, J. R., \& Committee, A. I. H. (1990). Metals Handbook: Irons, Steels, and Highperformance Alloys. Properties and selection: ASM International.

Gaskell, D. R. (2008). Introduction to the Thermodynamics of Materials, Fifth Edition: Taylor \& Francis.

Gild, J., Zhang, Y., Harrington, T., Jiang, S., Hu, T., Quinn, M. C., . . Luo, J. (2016). HighEntropy Metal Diborides: A New Class of High-Entropy Materials and a New Type of Ultrahigh Temperature Ceramics. [Article]. Scientific Reports, 6, 37946.

Kattner, U. R. (1997). The thermodynamic modeling of multicomponent phase equilibria. [Review]. JOM, 49(12), 14-19.

Laura, S., \& Diletta, S. (2011). Densification of ZrB2-TaSi2 and HfB2-TaSi2 Ultra-HighTemperature Ceramic Composites. Journal of the American Ceramic Society, 94(6), 1920-1930.

Megaw, H. D. (1973). Crystal structures: a working approach: Saunders.

Mohanty, S., Samal, S., Tazuddin, A., Twari, C., Gurao, N., \& Biswas, K. (2015). Effect of processing route on phase stability in equiatomic multicomponent Ti20Fe20Ni20Co20Cu20 high entropy alloy. Materials Science and Technology, 31, 10.

Murty, B. S., Yeh, J. W., \& Ranganathan, S. (2014). Chapter 1 - A Brief History of Alloys and the Birth of High-Entropy Alloys High Entropy Alloys (pp. 1-12). Boston: Butterworth-Heinemann.

Navrotsky, A., \& Kleppa, O. J. (1967). The thermodynamics of cation distributions in simple spinels. Journal of Inorganic and Nuclear Chemistry, 29(11), 2701-2714. 
Rost, C. M., Sachet, E., Borman, T., Moballegh, A., Dickey, E. C., Hou, D., . . Maria, J.-P. (2015). Entropy-stabilized oxides. [Article]. Nature Communications, 6, 8485.

Sharma, A. S., Yadav, S., Biswas, K., \& Basu, B. (2018). High-entropy alloys and metallic nanocomposites: Processing challenges, microstructure development and property enhancement. Materials Science and Engineering: R: Reports, 131, 1-42.

Tazuddin, Biswas, K., \& Gurao, N. P. (2016). Deciphering micro-mechanisms of plastic deformation in a novel single phase fcc-based $\mathrm{MnFeCoNiCu}$ high entropy alloy using crystallographic texture. [Article]. Materials Science and Engineering A, 657, 224233.

Tazuddin, Gurao, N. P., \& Biswas, K. (2017). In the quest of single phase multi-component multiprincipal high entropy alloys. [Article]. Journal of Alloys and Compounds, 697, 434-442.

Yeh, J. W., Chen, S. K., Lin, S. J., Gan, J. Y., Chin, T. S., Shun, T. T., . . Chang, S. Y. (2004). Nanostructured High-Entropy Alloys with Multiple Principal Elements: Novel Alloy Design Concepts and Outcomes. Advanced Engineering Materials, 6(5), 299303.

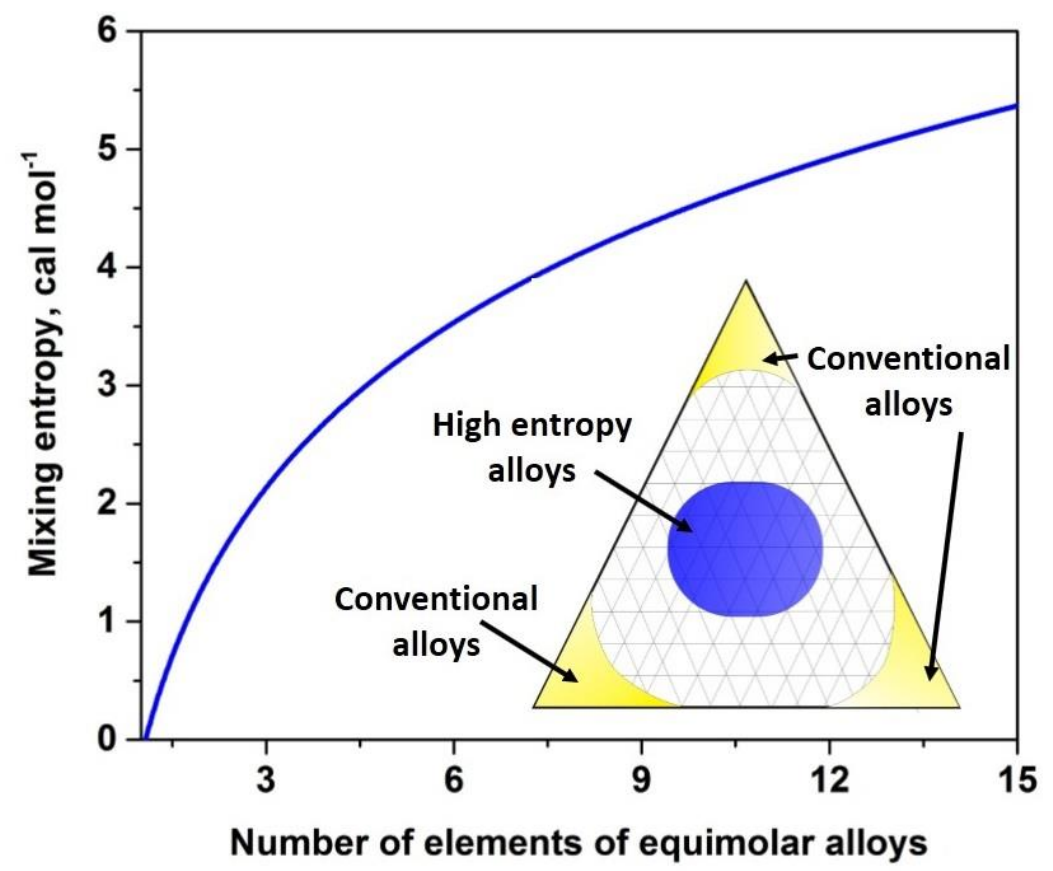

(a) 


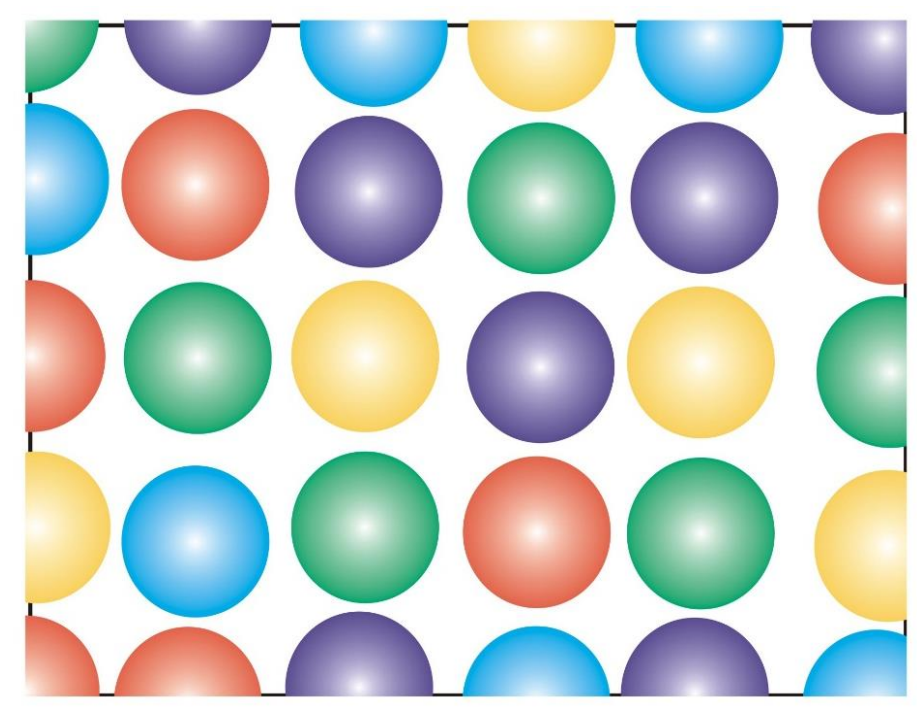

(b)

Figure 1: (a) Entropy change of mixing $\left(\Delta S_{m i x}\right)$ as a function of number of elements $(N)$ (b) HEA alloys lattice consisting more elements $(N>5)$ atom.

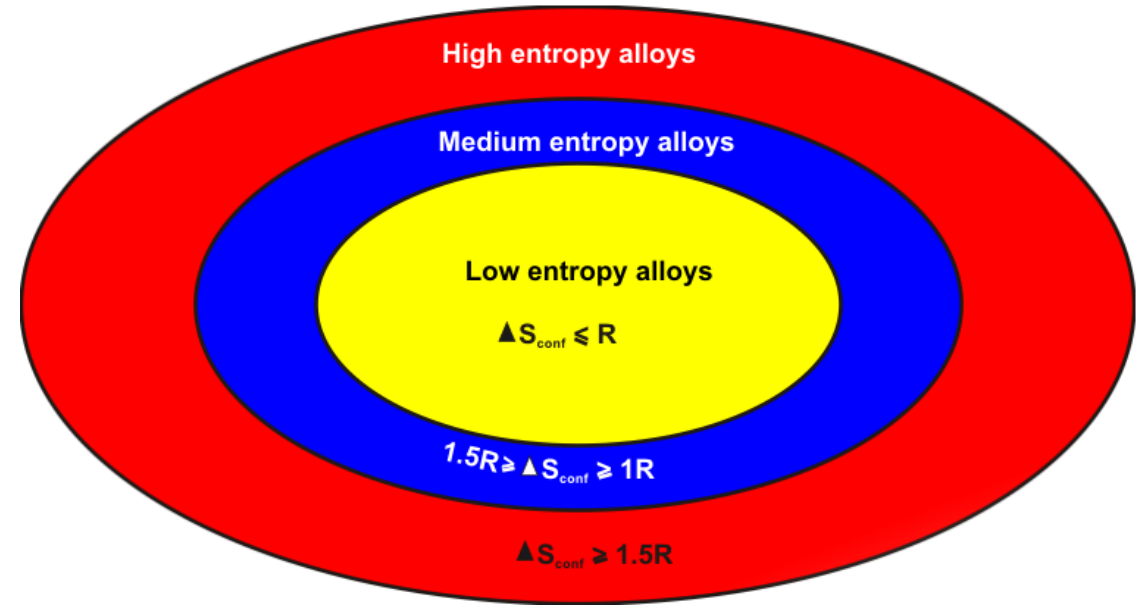

Figure 2: Alloy world as function of configurational entropy 
binary

temary

quaternary

$$
G=\sum X_{i} G_{i}^{0}+R T \sum x_{i} \ln x_{i}+G^{e x}
$$

Assessment: $G_{\text {bin }}^{e x}$

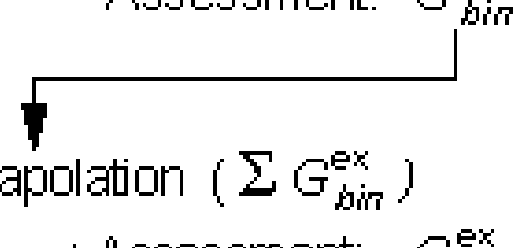

+Assessment: $G^{\text {ex }}$

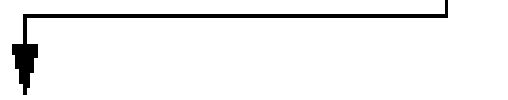

Extrapolation ( $\Sigma G_{\text {bir }}^{e x}+\sum G_{\text {tar }}^{e x}$ )

+Assessment: $G_{q u a}^{e x}$ 
(a)

FCC1(MnFeCoNiCu)

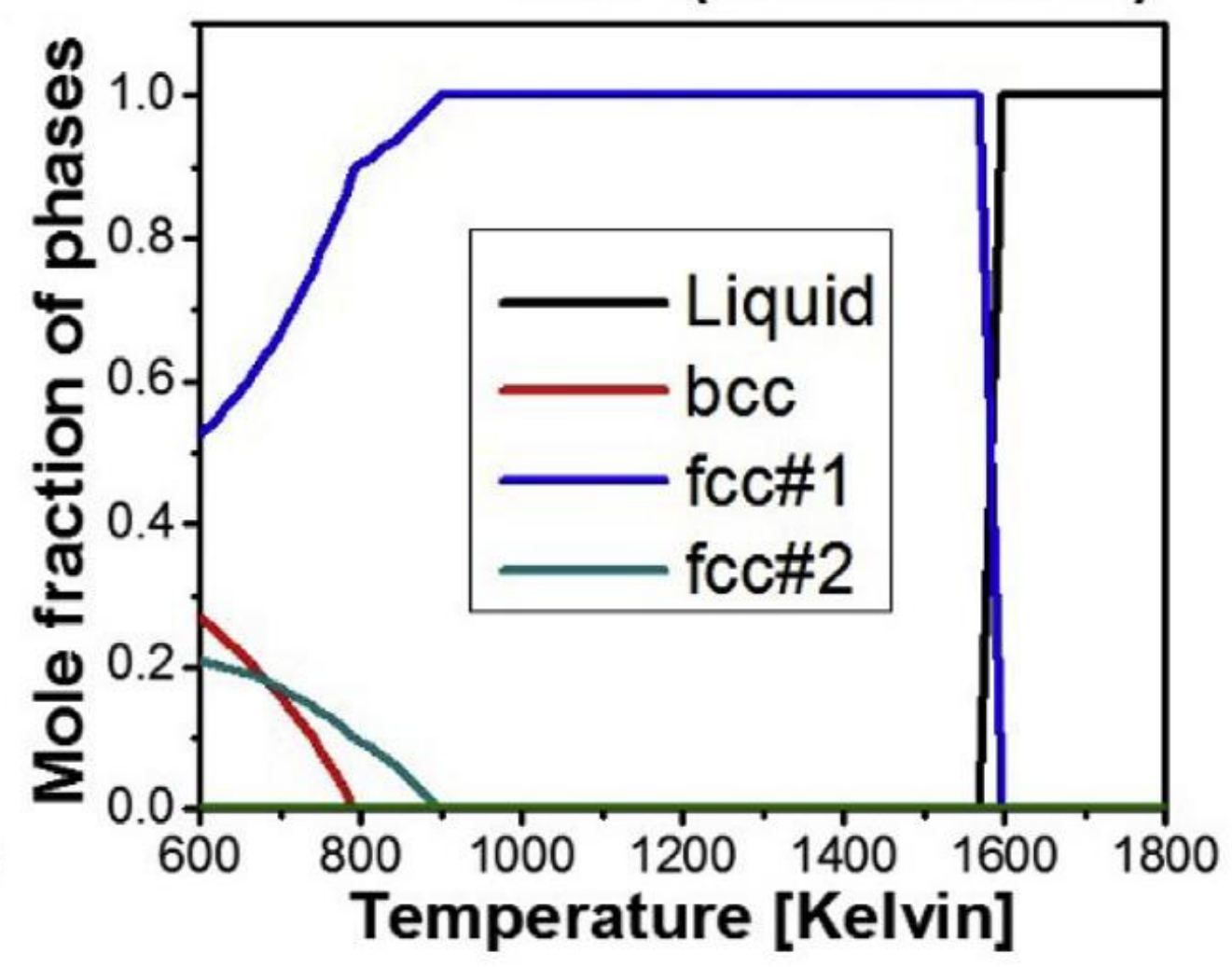

(b)

Figure 3: (a) CALPHAD methodology. The Gibbs energies of the constituent sub-systems for extrapolation to higher element alloys system (Kattner, 1997) (b) Phase fraction as function of Temperature.(Tazuddin et al., 2017)(permission from elsevier). 


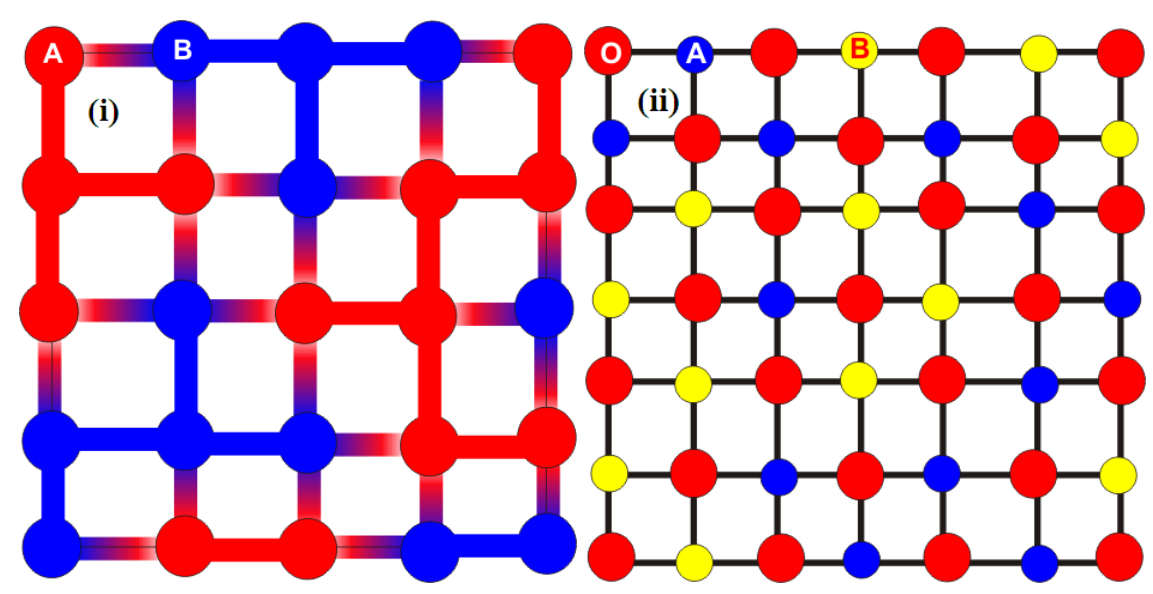

(a)

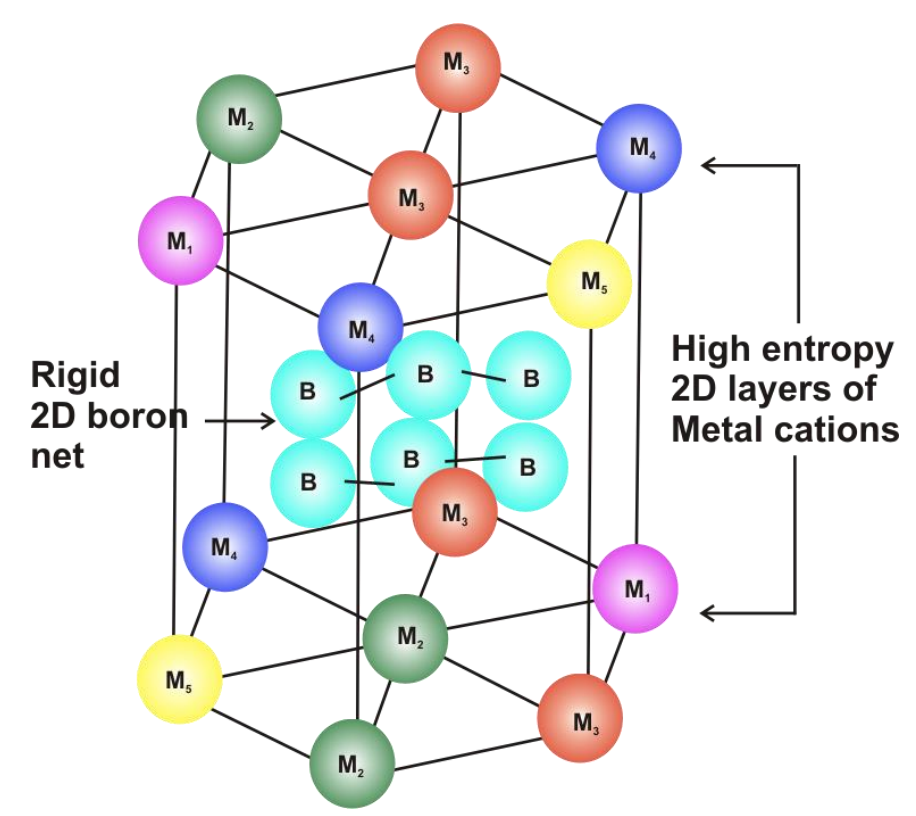

(b)

Figure 4: (a) Binary metallic compared with a ternary oxide. A schematic shows of two lattices describing how the first-near-neighbour environments between species having different electronegativity (the darker the more negative charge localized) for (i) a random binary metal alloy and (ii) random pseudo-binary mixed oxide (Rost et al., 2015). (b) Schematic of the atomic arrangement (layered hexagonal crystal structure) of the high-entropy metal diborides. $\left(\mathrm{M}_{1}, \mathrm{M}_{2}, \mathrm{M}_{3}, \mathrm{M}_{4}\right.$, and $\mathrm{M}_{5}$ represent five 
different transition metals high-entropy materials), with mixed ionic and covalent (M-B) bonds between the metals and boron (Gild et al., 2016).

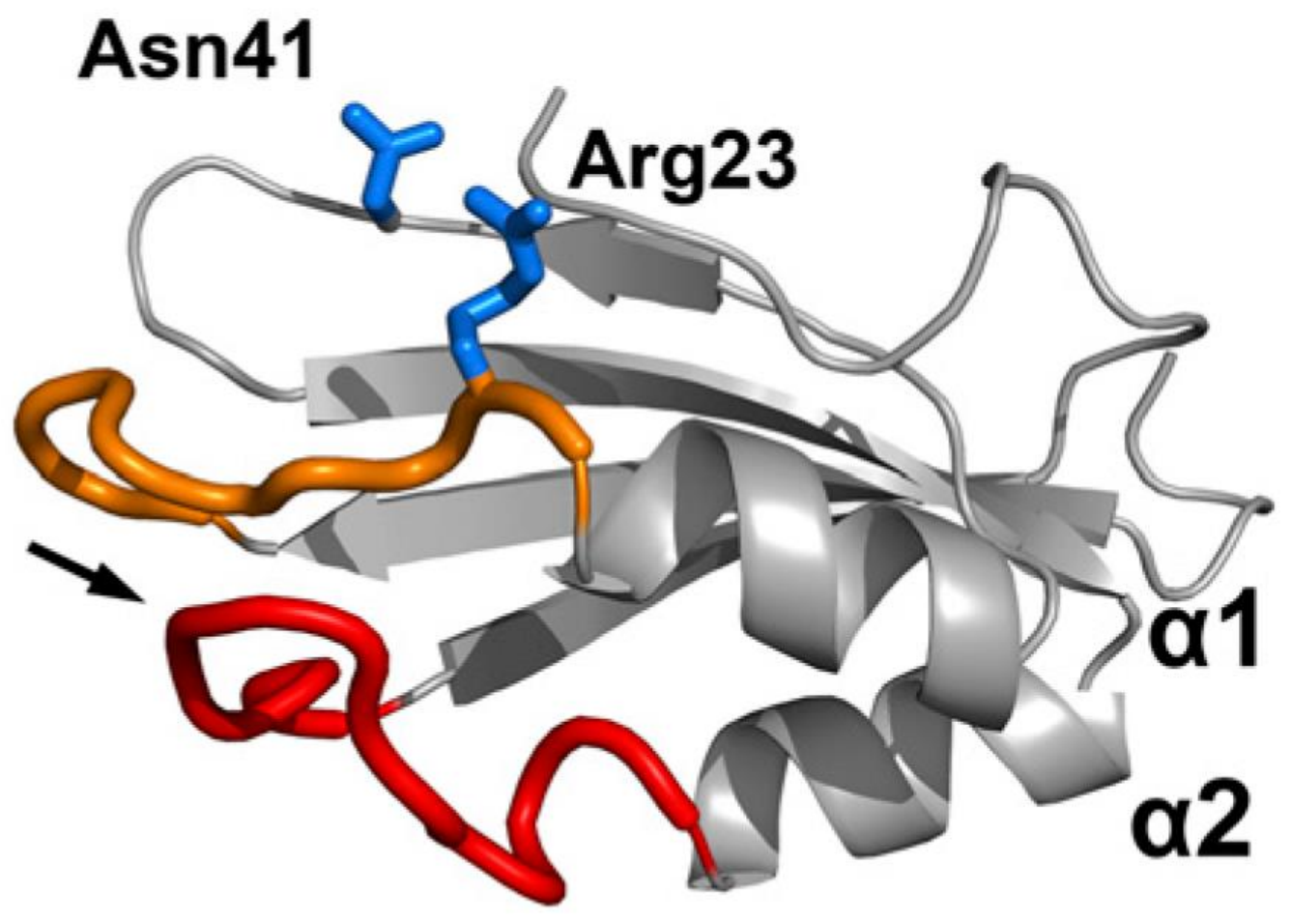

Figure 5: Model of hmAcP, constructed based on the solution structure of horse muscle AcP (Protein Data Bank ID code 1APS). (Dagan et al., 2013)(Permission from PNAS). 
